\title{
A Novel Readout System for Wireless Passive Pressure Sensors
}

\author{
Huixin $\mathrm{ZHANG}^{1,2}$, Yingping $\mathrm{HONG}^{1,2}$, Binger $\mathrm{GE}^{1,2}$, \\ Ting $\mathrm{LIANG}^{1,2}$, and Jijun XIONG ${ }^{1,2^{*}}$
}

\author{
${ }^{1}$ Key Laboratory of Instrumentation Science \& Dynamic Measurement (North University of China), Ministry of \\ Education, Taiyuan, 030051, China \\ ${ }^{2}$ Ministry of Education and Science Technology on Electronic Test \& Measurement Laboratory, Department of \\ Electronic Science and technology, North University of China, Taiyuan, 030051, China \\ *Corresponding author: Jijun XIONGＥ-mail: xiongjijun@nuc.edu.cn
}

\begin{abstract}
This paper presents a novel readout system for wireless passive pressure sensors based on the inductively coupled inductor and cavity (LC) resonant circuits. The proposed system consists of a reader antenna inductively coupled to the sensor circuit, a readout circuit, and a personal computer (PC) post processing unit. The readout circuit generates a voltage signal representing the sensor's capacitance. The frequency of the reader antenna driving signal is a constant, which is equal to the sensor's resonant frequency at zero pressure. Based on mechanical and electrical modeling, the pressure sensor design based on the high temperature co-fired ceramic (HTCC) technology is conducted and discussed. The functionality and accuracy of the readout system are tested with a voltage-capacitance measurement system and demonstrated in a realistic pressure measurement environment, so that the overall performance and the feasibility of the readout system are proved.
\end{abstract}

Keywords: Wireless passive pressure sensors, LC resonant circuits, HTCC, inductive coupling

Citation: Huixin ZHANG, Yingping HONG, Binger GE, Ting LIANG, and Jijun XIONG, "A Novel Readout System for Wireless Passive Pressure Sensors," Photonic Sensors, 2014, 4(1): 70-76.

\section{Introduction}

In some harsh environment applications, it is almost impractical to connect the pressure sensitive element to the conditioning electronics by the standard cables to measure the pressure, for example, in the automotive or biomedical field when the packages are inside the human body or upon rotating systems, for example in the industrial field when the harsh environment is incompatible with electronics. Therefore, sensors capable of measuring the pressure in harsh environments are needed. Inductor and cavity (LC) resonant sensors can represent valid solutions since they do not require a wiring liking and power supply. The sensors are composed of a capacitance cavity which is sensitive to the external pressure and a planar spiral inductor which is connected to the capacitance to form an LC resonant circuit, and they have been widely used in many applications [1-4].

Some measurement methods about the capacitance change in the LC pressure sensors were reported. As an example, impedance or network analyzers are used as readout devices for the resonance sensors [5, 6]. Although these devices are accurate and adaptable, they are expensive and bulky, in addition, they restrict the use of this

Received: 25 November 2013 / Revised version: 10 December 2013

(C) The Author(s) 2013. This article is published with open access at Springerlink.com

DOI: $10.1007 / \mathrm{s} 13320-013-0155-\mathrm{y}$

Article type: Regular 
measurement method outside the laboratory environment. Other options are devices that sweep over the frequency range, measuring the impedance amplitude, phase, real part, and imaginary part [7-9]. Some researchers have proposed a readout system which is based on coherent demodulation for mapping the input impedance seen from the reader antenna side to a direct current (DC) output voltage in order to get the sensor resonance frequency [10]. In a humidity measurement application, Marioli et al. have demonstrated a measurement distance compensation for determining the capacitance in the inductively coupled resonance sensor [11]. Due to the resonance frequency of the resonant sensor changes within a certain range, the testing methods which are based on measuring the resonance frequency all need to provide the linear or exponential sweep frequency analog signal source, and the frequency response performance of the testing system is greatly reduced in a frequency sweep cycle, then affects the testing results. At the same time, the measurement accuracy also heavily depends on the output precision of the signal source and the sampling rate of the readout circuit. In this paper, a novel readout system for the wireless passive pressure sensor capable of operating in harsh environments for pressure monitoring is proposed. The frequency of the reader antenna driving signal needed in the readout system is a certain value which is equal to the sensor's resonant frequency at zero pressure, and the pressure information contained in the sensor's capacitance is detected by the readout system circuit and then transmitted to computer. A wireless passive pressure sensor based on the high temperature co-fired ceramic (HTCC) technology is realized, and the variation of the sensor's capacitance caused by the pressure applied is detected by a designed wireless pressure sensing system. This system realizes the test of the sensor without the network analyzer or network analyzer, and all could contribute to the integration and promotion of the wireless passive pressure sensors.

\section{Sensor design and analysis}

The remote powering and wireless interrogation is obtained through an inductively powered system. As shown in Fig. 1, an inductive link between the sensor's inductor and the reader antenna is used to power the LC passive pressure sensor, therefore, allowing its application where the battery of the sensor cannot be recharged or exchanged. The pressure information in terms of the sensor's capacitance is transmitted into the reader side. When the sensitive capacitor of the LC resonant circuit feels the external pressure, the capacitance varies, which leads to the change in the voltage signal across the terminals of the reader antenna, and the voltage variations in the sensor's response can be detected by the readout circuit.

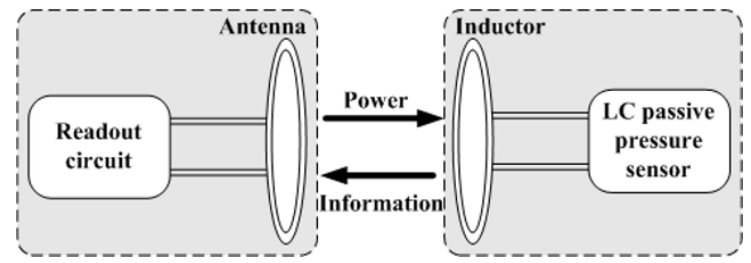

Fig. 1 Basic theory of wireless communication.

The LC passive pressure sensor, as demonstrated in Fig. 2, is composed of a ceramic multi-layer capacitor and a plane spiral inductor. The sensor prototype is designed and fabricated based on the HTCC. The structure is stacked in three layers, and the surface of the first ceramic layer is the inductor coil, which is designed as a square spiral type. The capacitor is a parallel plate type with round electrodes. The upper and lower capacitor electrodes are respectively placed on the surface of the first and the third layers. Electrical connection between the capacitor plates and inductor is realized with the electrical lead. Tape layers with the capacitor electrodes act as sensor membranes which react to the pressure. In order to ensure the sensor is completely gastight in harsh environments, the sensor is designed without the evacuation channel and exit hole. 


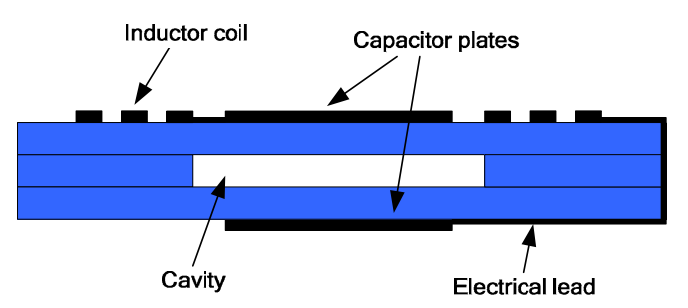

Fig. 2 Cross-section of the sensor.

The sensor's electromechanical model is presented in Fig. 3. When the sensitive membranes react to the pressure, the round sensitive membrane has a central deflection $d_{0}$ as given in [12], and the sensor capacitance can be derived using the expression [13]

$$
C=C_{0} \times \tanh ^{-1}\left(\sqrt{\frac{2 d_{0}}{t_{g}+\frac{2 t_{m}}{\varepsilon_{r}}}}\right) / \sqrt{\frac{2 d_{0}}{t_{g}+\frac{2 t_{m}}{\varepsilon_{r}}}}
$$

where $t_{g}$ stands for the cavity thickness, $t_{m}$ is the thickness of the sensitive membrane, $\varepsilon_{0}$ and $\varepsilon_{r}$ represent the dielectric constant of air and relative dielectric constant, respectively, and $C_{0}$ is the capacitance at zero pressure, which is given by[13]

$$
C_{0}=\frac{\varepsilon_{0} \pi a^{2}}{t_{g}+\frac{2 t_{m}}{\varepsilon_{r}}}
$$

Here, $a$ is the radius of the round electrode.

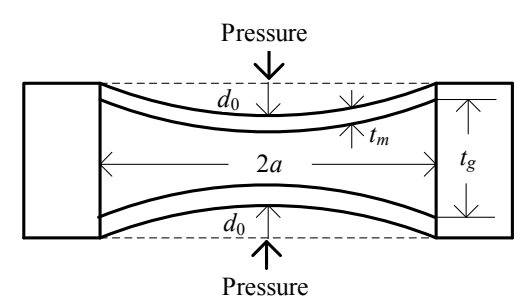

Fig. 3 Sensor's electromechanical model.

Using the Ferro A6 raw ceramic as the basal material and Ferro 3116 silver paste for the silk-screen printing, the sensor sample is completed. The relevant geometrical parameters of the inductor and the capacitor are given in Tables 1 and 2, respectively. The sensor's theoretical capacitance at zero pressure $C_{0}$ is $3.54 \mathrm{pF}$. The square planar spiral inductance can be derived from the following expression [14]:

$$
L=\frac{k_{1} \mu_{0} n^{2} d_{\text {avg }}}{1+k_{2} \rho}
$$

where coefficients $k_{1}=2.34$ and $k_{2}=2.75$ are layout dependent, $\mu_{0}$ is the permeability of vacuum, $d_{\text {avg }}$ and $\rho$ are the average diameter and the fill ratio, respectively, which are given by

$$
\begin{gathered}
d_{\text {avg }}=\frac{d_{\text {out }}+d_{\text {in }}}{2} \\
\rho=\frac{d_{\text {out }}-d_{\text {in }}}{d_{\text {out }}+d_{\text {in }}} .
\end{gathered}
$$

Then, the resonance frequency of the sensor can be derived from the following expression:

$$
f_{0}=\frac{1}{2 \pi \sqrt{L C}} .
$$

Table 1 Geometrical parameters of the inductor designed.

\begin{tabular}{cc}
\hline Parameter & Value \\
\hline Inner diameter of the inductor coil $d_{\text {in }}$ & $12 \mathrm{~mm}$ \\
Outer diameter of the inductor coil $d_{\text {out }}$ & $35 \mathrm{~mm}$ \\
Width of the inductor coil $L_{w}$ & $0.5 \mathrm{~mm}$ \\
Spacing between adjacent segments $L_{s}$ & $0.5 \mathrm{~mm}$ \\
Number of the inductor coil $n$ & 15 \\
\hline
\end{tabular}

Table 2 Geometrical parameters of the capacitor designed.

\begin{tabular}{cc}
\hline Parameter & Value \\
\hline Radius of the round electrode $a$ & $4 \mathrm{~mm}$ \\
Thickness of the sensitive membrane $t_{m}$ & $100 \mu \mathrm{m}$ \\
Cavity thickness $t_{g}$ & $100 \mu \mathrm{m}$ \\
\hline
\end{tabular}

\section{Readout system}

\subsection{Electrical model and simulation}

The proposed readout system consists of a reader antenna, a readout circuit, and a PC post processing unit. The electrical equivalent circuit is illustrated in Fig. 4. Here, $U_{1}$ is a sinusoidal signal (the reader antenna driving signal) with a certain frequency which is equal to the sensor's resonant frequency at zero pressure, $R_{1}$ and $R_{2}$ indicate the self-resistances of the reader antenna and the sensor's circuit, $C_{1}$ and $C_{2}$ are the capacitances of the reader's side and the sensor, respectively, and $M$ indicates the mutual inductance of the coupled inductor coils. 


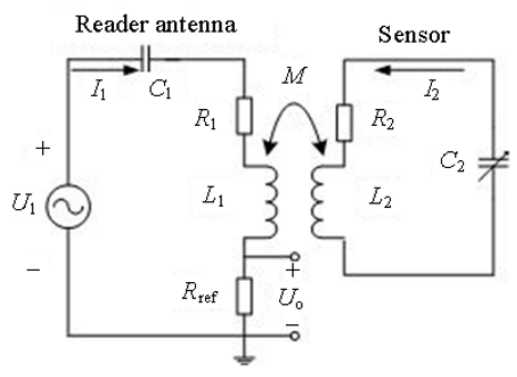

Fig. 4 Equivalent circuit diagram of the readout system circuit.

The following equations can be derived using Kirchhoff's law:

$$
\left\{\begin{array}{c}
\left(R_{1}+j 2 \pi f L_{1}+\frac{1}{j 2 \pi f C_{1}}+R_{\mathrm{ref}}\right) I_{1}+j 2 \pi f M I_{2}=U_{1} \\
j 2 \pi f M I_{1}+\left(R_{2}+j 2 \pi f L_{2}+\frac{1}{j 2 \pi f C_{2}}\right) I_{2}=0
\end{array}\right.
$$

where $f$ is the frequency of the sinusoidal signal, using the substitutions

$$
\begin{gathered}
Z_{1}=R_{1}+j 2 \pi f L_{1}+\frac{1}{j 2 \pi f C_{1}}+R_{\mathrm{ref}} \\
Z_{2}=R_{2}+j 2 \pi f L_{2}+\frac{1}{j 2 \pi f C_{2}} \\
Z_{M}=j 2 \pi f M .
\end{gathered}
$$

Equations (7) and (8) can be simplified as

$$
\left\{\begin{array}{l}
Z_{1} I_{1}+Z_{M} I_{2}=U_{1} \\
Z_{M} I_{1}+Z_{2} I_{2}=0 .
\end{array}\right.
$$

The output voltage signal $U_{\mathrm{o}}$ across the resistance $R_{\text {ref }}$ can be derived by solving (12) and (13) as

$$
U_{\mathrm{o}}=\frac{R_{\mathrm{ref}}}{Z_{1}+(2 \pi f M)^{2} Y_{2}} U_{1} \text {. }
$$

The amplitude of the sinusoidal signal $U_{1}$ is $A$, when the voltage of the sinusoidal signal is the maximum, the following equation can be derived:

$$
U_{m}=\frac{R_{\mathrm{ref}}}{Z_{1}+(2 \pi f M)^{2} Y_{2}} A .
$$

Then, when the voltage of the sinusoidal signal is the maximum, the magnitude of the output voltage signal $\left|U_{m}\right|$ is

$$
\left|U_{m}\right|=\left|\frac{R_{\mathrm{ref}}}{Z_{1}+(2 \pi f M)^{2} Y_{2}} A\right| .
$$

Equations (9), (10), and (14) show that the output voltage signal is related to the sensor's capacitance $C_{2}$. Figure 5 shows the simulation result using the
MATLAB, with the parameter values summarized in Table 3. It can be seen that the magnitude of the output voltage signal $\left|U_{m}\right|$ is the minimum when the sensor's capacitance is $20 \mathrm{pF}$, where the sensor's resonant frequency is equal to the sinusoidal signal's frequency. The pressure information contained in the sensor's capacitance can be obtained from the output voltage signal. The output voltage signal can be analyzed and processed by the readout circuit and the PC post processing unit to get the sensor's capacitance.

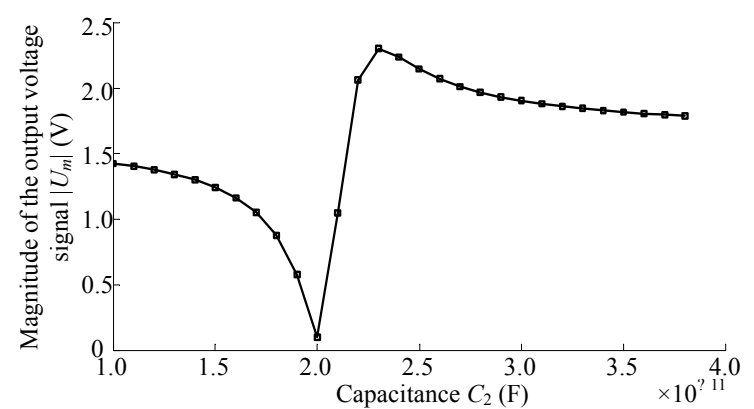

Fig. 5 Magnitude of the output voltage signal $\left|U_{m}\right|$ vs. the pressure sensor's capacitance.

Table 3 System parameters.

\begin{tabular}{cc}
\hline Parameter & Value \\
\hline Reader inductance $L_{1}$ & $4182 \mathrm{nH}$ \\
Sensor inductance $L_{2}$ & $4182 \mathrm{nH}$ \\
Frequency of the sinusoidal signal $f$ & $17.4 \mathrm{MHz}$ \\
Resonance frequency of the sensor at zero pressure $f_{0}$ & $17.4 \mathrm{MHz}$ \\
Capacitance of the reader's side $C_{l}$ & $20 \mathrm{pF}$ \\
Coupling distance $d$ & $20 \mathrm{~mm}$ \\
\hline
\end{tabular}

\subsection{Instrumentation}

The measurement instrumentation (as shown in Fig. 6) used in this work includes the readout circuit, the reader antenna, the LC passive pressure sensor, and the sensor dummy. The printed circuit board (PCB) of the designed readout circuit is shown in Fig. 7(b). The sinusoidal signal with a certain frequency and the output voltage signal across the resistance $R_{\text {ref }}$ are multiplied using the Gilbert cell-based mixer AD831, and then a low-pass filter circuit is used to filter the mixer's output signal in a DC output voltage. A fast 16-bit ADC (AD7667) is used to convert the DC output voltage into the 
digital form.

The readout circuit also contains a microcontroller unit CY7C68013 for communicating with the PC via a USB interface. The PC post-processing software analyzes and processes the digital data, calculates and saves the sensor's capacitance.

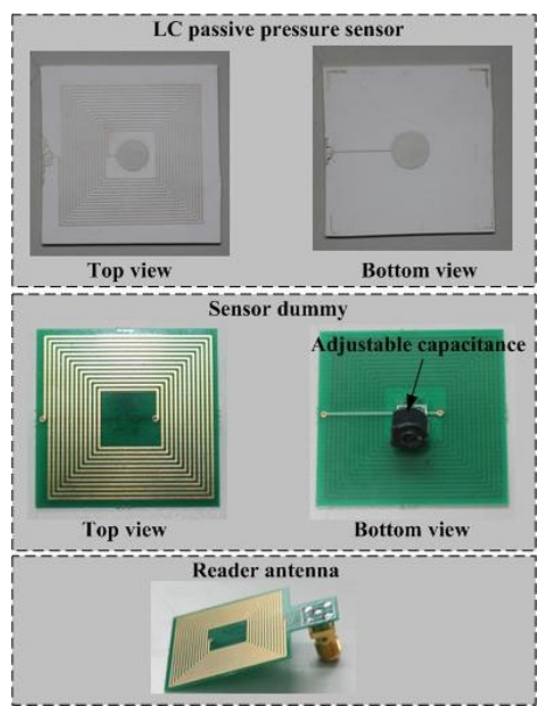

(a) Sensors and reader antenna

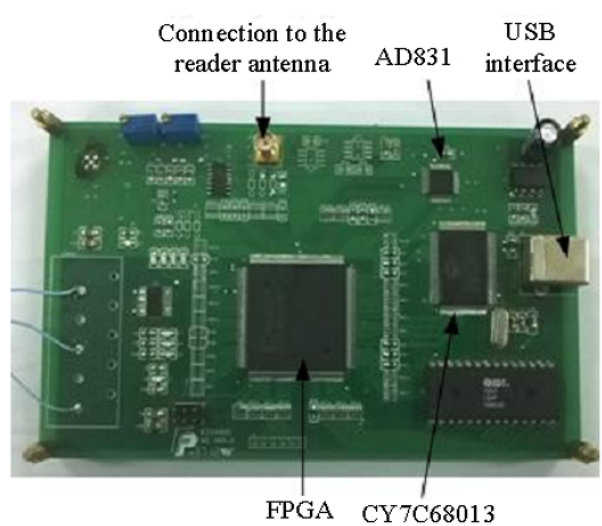

(b) Designed readout circuit

Fig. 6 Instrumentation used in this work.

\section{Experiments}

A voltage-capacitance measurement system which consisted of the readout instrumentation, pressure sensor dummy, coupling distance testing platform, and computer as shown in Fig. 7 was designed. The readout circuit and the sinusoidal signal source circuit were encapsulated in the readout instrumentation. The reader antenna and the sensor dummy were a certain distance apart without any electric connection, the coupling distance which could be measured by the platform shown in Fig. 7 is $20 \mathrm{~mm}$.

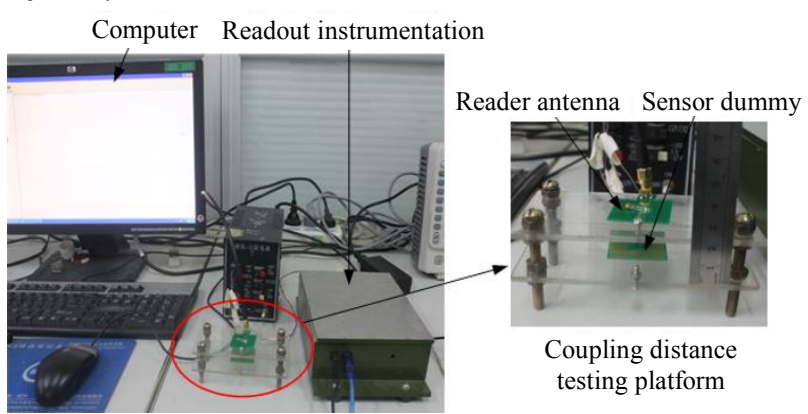

Fig. 7 A voltage-capacitance measurement system.

Experimental tests on the measurement system have been carried out by varying the sensing adjustable capacitance, with the parameter values summarized in Table 3, and the reader antenna inductor and the sensing inductor were designed identically. Figure 8 shows the measured output voltage of the readout circuit versus the sensing adjustable capacitance using an LC resonator as a sensor dummy inductively coupled to the reader antenna. We can see that the tendency of the measured output voltage is in good accordance with the simulation result. It proves the feasibility of the readout system.

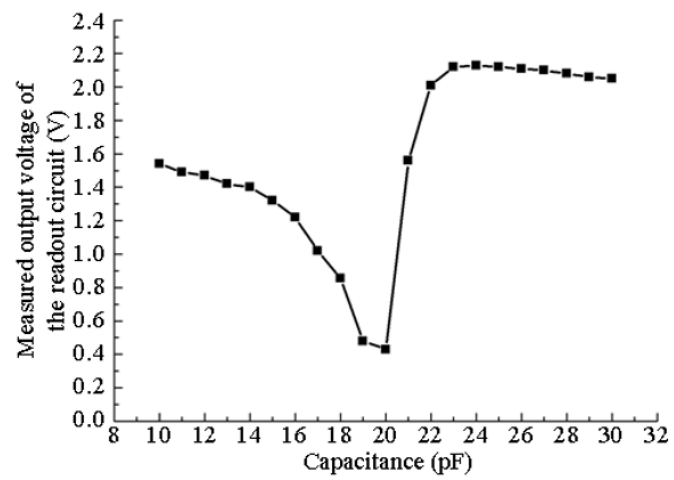

Fig. 8 Measured output voltage of the readout circuit vs. the sensing adjustable capacitance.

The LC passive pressure sensor designed based on the HTCC has been tested as a function of the pressure from 0 bar to 2 bar with the wireless pressure sensing system consisting of the pressure controller, readout instrumentation, pressure vessel, 
and computer shown in Fig. 9. The reader antenna and sensor were put into the pressure vessel, where nitrogen gas was pumped through GE PACE 6000 pressure controller to make sure of a controllable uniform pressure condition. The reader antenna was connected to the readout instrumentation through test ports of the pressure vessel. The frequency of sinusoidal signal was set as $32.9 \mathrm{MHz}$, which was equal to the measured resonant frequency of the sensor at zero pressure.

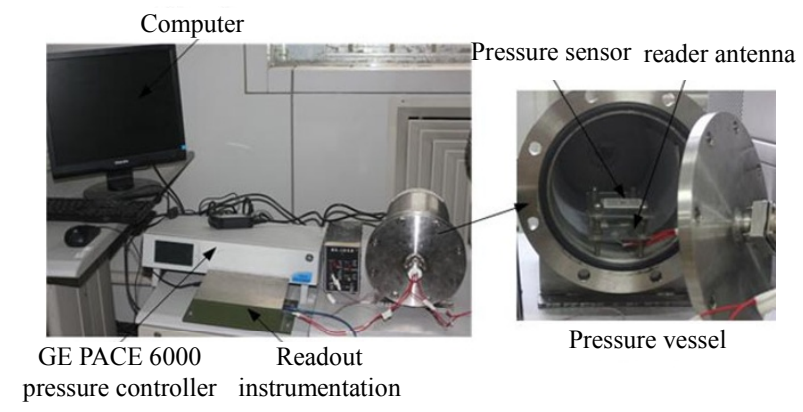

Fig. 9 Wireless pressure sensing system.

From the measurement results shown in Figs. 10 and 11, it is clear that the measured output voltage of the readout circuit increased as the pressure increased from 0 bar to 2 bars. The tested sensor's capacitance at zero pressure was $3.8 \mathrm{pF}$, which was $7.34 \%$ larger than the theoretical value $(3.54 \mathrm{pF})$. The discrepancy of the sensor's capacitance is mainly caused by the possible factors concluded as following: (1) large cavity dimensions cause the collapse of the membrane, the distance between electrodes become small, finally the capacitance becomes larger; (2) the capacitor electrodes are larger than the cavity. The superfluous parts of the electrodes, which were sandwiched between ceramic layers but not the ceramic layer and vacuum as the overlap parts, distributed a lot to the sensor's capacitance because of the larger dielectric constant of the ceramic compared to the vacuum [15]. Both effects would increase the capacitance. In addition, the measurement results may be influenced by the surrounding environment and the measurement setup.

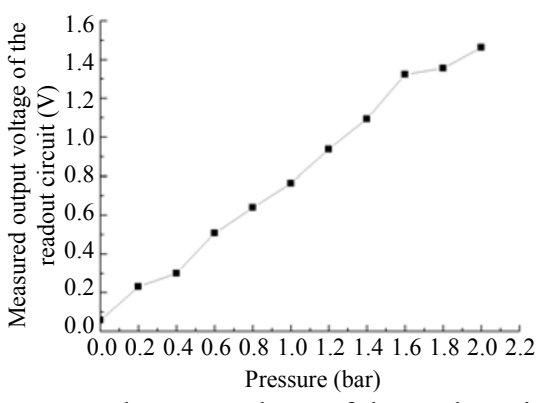

Fig. 10 Measured output voltage of the readout circuit vs. the applied pressure.

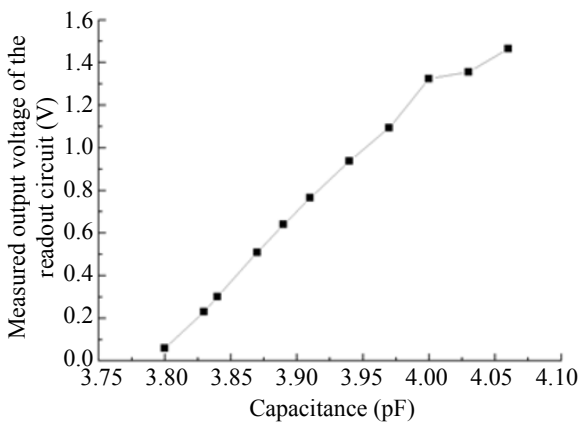

Fig. 11 Measured output voltage of the readout circuit vs. the pressure sensor's capacitance.

\section{Conclusions}

In this paper, a novel readout system for wireless passive pressure sensors capable of operating in the harsh environments is presented. An equivalent circuit diagram of the readout system circuit, relying on a voltage variation output, has also been introduced. It is demonstrated that the pressure information contained in the sensor's capacitance can be obtained from the readout system circuit's output voltage signal.

The readout system has been tested by a voltage-capacitance measurement system, and the measured results were in good accordance with the simulation results, which proved the feasibility of the readout system. In addition, a wireless passive pressure sensor designed based on the HTCC was tested in a pressure vessel. The proposed readout system is practical, effective, and the overall performance of it justifies the further studies. In the pressure tested application, the readout system will give an advantage over the earlier systems, because the earlier systems which are based on measuring 
the resonance frequency all need to provide the linear or exponential sweep frequency analog signal source, and the frequency response performance of the testing system is greatly reduced in a frequency sweep cycle, then affects the testing results. The compact hardware of the readout system can also be utilized in other portable applications, since the developed device can be used instead of the impedance or network analyzers as a readout device for $\mathrm{LC}$ resonant sensors. The readout system makes it possible to take the pressure measurements out of the laboratory, which will endorse the development of wireless passive pressure sensors.

\section{Acknowledgment}

The research was supported by the National Basic Research Program of China (973 Program) under the grant of NO. 2010CB334703, additionally, the research was also supported by National Natural Science Foundation of China under grant of NO.51075375.

Open Access This article is distributed under the terms of the Creative Commons Attribution License which permits any use, distribution, and reproduction in any medium, provided the original author(s) and source are credited.

\section{References}

[1] M. A. Fonseca, J. M. English, M. von Arx, and M. G. Allen, "Wireless micromachined ceramic pressure sensor for high-temperature applications," Journal of Microelectromechanical Systems, 2002, 11(4): 337-343.

[2] E. D. Birdsell, J. Park, and M. G. Allen, "Wireless ceramic sensors operating in high temperature environments," 40th AIAA/ASME/SAE/ASEE Joint Propulsion Conference, 2004, July 11-14, pp. 1-10.

[3] E. D. Birdsell and M. G. Allen, "Wireless chemical sensors for high temperature environments," Solid-State Sensors, Actuators, and Microsystems Workshop, Hilton Head Island, South Carolina, 2006, June 4-8, pp. 212-215.
[4] T. J. Harpster, S. Hauvespre, M. R. Dokmeci, and K. Najafi, "A passive humidity monitoring system for in situ remote wireless testing of micropackages," Journal of Microelectromechanical Systems, 2002, 11(1): 61-67.

[5] P. J. Chen, S. Saati, R. Varma, M. S. Humayun, and Y. C. Tai, "Wireless intraocular pressure sensing using microfabricated minimally invasive flexible-coiled LC sensor implant," Journal of Microelectromechanical Systems, 2010, 19(4): 721-734.

[6] V. Sridhar and K. Takahata, "A hydrogel-based passive wireless sensor using a flexcircuit inductive transducer," Sensors and Actuators A: Physical, 2009, $155(1), 58-65$.

[7] T. Salpavaara, J. Verho, P. Kumpulainen, and J. Lekkala, "Readout methods for an inductively coupled resonance sensor used in pressure garment application," Sensors and Actuators A: Physical, 2011, 172(1): 58-65.

[8] C. C. Collins, "Miniature passive pressure transensor for implanting in the eye," IEEE Transactions on Biomedical Engineering, 1967, BME-14(2): 74-83.

[9] Sajeeda and T. J. Kaiser, "Passive telemetric readout system," IEEE Sensors Journal, 2006, 6(5): 1340-1345.

[10] R. Nopper, R. Has, and L. Reindl, “A wireless sensor readout system $\square$ circuit concept, simulation, and accuracy," IEEE Transactions on Instrumentation and Measurement, 2011, 60(8): 2976-2983.

[11] D. Marioli, E. Sardini, M. Serpelloni, and A. Taroni, "A distance compensated telemetric humidity sensor based on the parasitic capacitance variation," Proc. of the IEEE Instrumentation and Measurement Technology Conference, 2008, pp. 655-660.

[12] S. Timoshenko and S. Woinowsky-Krieger, Theory of Plates and Shells (2nd Edition). London, UK: McGraw Hill, 1959.

[13] M. A. Fonseca, "Polymer/ceramic wireless MEMS pressure sensors for harsh environments: high temperature and biomedical application," Ph.D. Dissertation, School of Electrical and Computer Engineering, 2007.

[14] S. S. Mohan, M. Del Mar Hershenson, S. P. Boyd, and T. H. Lee, "Simple accurate expressions for planar spiral inductances," IEEE Journal of Solid-state Circuits, 1994, 34(10): 1419-1424.

[15] J. J. Xiong, Y. Li, Y. P. Hong, B. Z. Zhang, T. H. Cui, Q. L. Tan, et al., "Wireless LTCC-based capacitive pressure sensor for harsh environment," Sensors and Actuators A: Physical, 2013, 197: 30-37. 